\title{
A Mini-review of the 2019 Novel Coronavirus, SARS-CoV-2
}

\author{
David Rutenberg* and Yumeng Zhang \\ Department of Internal Medicine, University of South Florida, USA
}

*Corresponding author: David Rutenberg, Department of Internal Medicine, University of South Florida, 17 Davis Blvd, Suite 308, Tampa, FL 33606, USA.

To Cite This Article: David Rutenberg, A Mini-review of the 2019 Novel Coronavirus, SARS-CoV-2. 2020 - 8(1). AJBSR.MS.ID.001226.

DOI: 10.34297/AJBSR.2020.08.001226.

Received: 海 February 21, 2020; Published: 眥 March 10, 2020

\begin{abstract}
A novel coronavirus has recently emerged in December 2019 out of Wuhan City, Hubei Province, China. The virus can cause disease, termed coronavirus virus disease 2019, or COVID-19. Its presentation is similar to severe acute respiratory syndrome, known as SARS, because of the disease manifesting as mild-to-severe respiratory symptoms. Animals sold out of a seafood market in Wuhan are thought to have transmitted the virus. Since its discovery, it has infected more than 78,000 people and spread to at least 30 other countries. The majority of cases are in China, but cases are increasing in multiple countries throughout the world, including the United States. The virus shares many similar physical and pathogenic features as the virus that causes SARS and has thus been named SARS-CoV-2. Close contact can cause transmission of the virus. Initial symptoms in 2-14 days of viral contact are similar to influenza, including fever, cough, myalgias, and dyspnea. Those with severe illness are generally older or those with medical comorbidities. There are currently no cures or vaccines against the virus.
\end{abstract}

In comparison to seasonal influenza, which is currently more prevalent than SARS-CoV-2, SARS-CoV-2 is considered more infectious and carries a higher mortality rate. There is still much to learn about the virus, as it is expected to continue spreading. Global efforts are being made via public health emergencies to identify those infected and quarantine them early on to prevent further spread.

Abbreviations: SARS-CoV-2: Severe Acute Respiratory Syndrome Coronavirus 2; COVID-19: Coronavirus Disease 2019; CoV: Coronavirus; SARS-CoV: Severe Acute Respiratory Syndrome Coronavirus; MERS-CoV: Middle Eastern Respiratory Syndrome Coronavirus; ACE2: Angiotensin-Converting Enzyme 2; RBD: Receptor Binding Domain; RBM: Receptor Binding Motif; RT-PCR: Reverse-transcription Polymerase Chain Reaction

\section{Introduction}

The 2019 novel coronavirus, previously known as 2019nCoV, now named SARS-CoV-2 by the International Committee on Taxonomy of Viruses, is a newly emerging virus affecting the respiratory tract. The disease the virus causes, which was named by the World Health Organization, is coronavirus disease 2019, better known as COVID-19. It has quickly spread internationally, affecting more than 78,000 people and claiming over 2,400 lives since its discovery in December 2019. It continues to be a global health concern, with multiple countries declaring public health emergencies, including the United States [1,2].

Coronavirus $(\mathrm{CoV})$ is an enveloped pathogen that contains a single-stranded positive-sense RNA that is $27-32 \mathrm{~kb}$ in length [3]. $\mathrm{CoV}$ is the largest known RNA virus [4]. It had gained notoriety in the medical and scientific communities within the last 20 years due to the outbreaks of severe acute respiratory syndrome CoV (SARS-CoV) in 2003 and Middle Eastern respiratory syndrome CoV (MERS-CoV) in 2012. SARS-CoV infected 8098 patients with a $10 \%$ mortality rate. MERS-CoV infected 2,254 patients with a $35 \%$ mortality rate [4]. The mortality rate of SARS-CoV-2 is currently estimated to be about 2\% [5]. Before these outbreaks, the majority of infections by $\mathrm{CoV}$ were found in birds and mammals [6].

There are four genera in the sub-family of Coronaviridae, and each genus has a preference to infect a specific type of host. Before the new outbreak, six CoVs cause infections in humans, the most serious, including SARS-CoV and MERS-CoV, and four others that cause mild upper and lower respiratory symptoms. Betacoronaviruses, including SARS-CoV, MERS-CoV, and now SARS$\mathrm{CoV}-2$, infect mammals, while deltacoronaviruses infect birds. Bats are believed to be the main reservoirs for beta-coronaviruses; other 
mammals, such as palm civets for SARS-CoV and dromedary camels for MERS-CoV, serve as an intermediate host. The crossing of the animal to humans, which has been documented in all three $\mathrm{CoV}$ outbreaks, makes CoV a zoonotic virus. [3,4,6].

SARS-CoV-2's physical structure and pathogenic behavior share similarities with SARS-CoV. CoVs utilize a protein called a spike (S) protein to bind to target cells and a cellular protease to prime the S protein. "The overall sequence similarities between [SARS-CoV-2] spike and SARS-CoV spike... are around 76\%-78\% for the whole protein, around $73 \%-76 \%$ for the RBD [receptor binding domain], and 50\%-53\% for the RBM [receptor binding motif]" [7]. The target cellular receptor for the $\mathrm{S}$ protein of SARS-CoV-2 was confirmed to be the same as SARS-CoV, angiotensin-converting enzyme 2 (ACE2). The cellular protease, TMPRSS2, was also the same [8] $[7,9]$. In spite of many similarities between SARS-CoV-2 and SARS$\mathrm{CoV}$, SARS-CoV-2 is more closely related to two other coronaviruses found in bats, bat-SL-CoVZC45, and bat-SL-CoVZXC21 [8], which suggests that bats are the reservoir for this virus [10].

SARS-CoV-2 was first discovered in Wuhan City, Hubei Province, China, during December 2019 after several patients developed viral pneumonia that appeared similar to SARS. The identified patients had similar exposure to the Huanan Seafood Wholesale Market in Wuhan, which sells live animals, including birds and other mammals. These mammals are postulated to have served as an intermediate host, similar to SARS-CoV and MERS-CoV, and transmitted the virus to humans, although the species of animal is still unknown. [11]. The virus has since spread to at least twentyfour countries [12].

The rapid spreading of disease has lead healthcare professionals and scientists to believe that the virus can be transmitted from person-to-person via close contact $[10,13]$. There is concern that the virus can be transmitted asymptomatically, prior to symptom onset [14]. The virus had been detected in respiratory secretions and stool, with respiratory secretions thought to be the primary means of transmission [15]. The incubation period from exposure to onset of symptoms ranges from 2 to 14 days [16]. An RT-PCR laboratory test kit testing upper and lower respiratory specimens are the method of detection [17]. In a small case series of 18 patients, radiographic findings on chest computed tomagraphy scan included ground-glass opacities with or without consolidation, opacities with a rounded appearance, and a crazy paving pattern. The distribution is typically bilateral involving multiple lobes of the lungs. Cavitary lung lesions, pleural effusions, lymphadenopathy, and pulmonary nodules were not found [18]. In a large case series published by JAMA, of 138 confirmed cases of COVID-19 in a Wuhan, China hospital, the most common symptoms included fever (98.6\%), fatigue (69.6\%), dry cough (59.4\%), myalgia (34.8\%), and dyspnea (31.2\%). The median age of those infected patients was 56 years old. Twenty-six percent of patients were admitted to the intensive care unit (ICU). The median age of those admitted to the ICU was generally older, 66 years, with the majority having other medical comorbidities. Of those infected, six died (4.3\%). At this time, there is no antibiotic or antiviral shown to improve the outcome of COVID-19 [10]. There is no vaccine to protect patients against SARS-CoV-2 [19]. International organizations recommended supportive therapies for the complications of COVID-19, including acute respiratory distress syndrome, septic shock, and prevention of other in-hospital adverse outcomes.

Questions often arise regarding prevention, mortality, and degree of contagiousness between the influenza virus and SARSCoV-2. Currently, the best way to prevent influenza and its complications is by vaccination, whereas there is no vaccine for SARS-CoV-2. The best way to prevent the spread of COVID-19 is to stay home if one feels sick, wash hands frequently, avoid touching one's own face, avoiding traveling to countries with endemic spread of the virus, and avoiding contact with those known to have the virus. According to the CDC, there has been an estimated 26 million cases of the flu and 14,000 related deaths, a $0.05 \%$ mortality rate, in the United States this season [20]. Globally, there are more than 78,000 cases of COVID-19 and over 2,400 related deaths, a mortality rate of about $2 \%$ [5]. Comparatively speaking to countries like China and Italy, the United States' spread is not yet as extensive [21]. Although the United States' outbreak is not yet as high, serious precaution must still be made, as COVID-19 has a mortality rate 40 times that of influenza. To compare the degree of contagiousness of each virus, the R0 of seasonal influenza is 1.3 [22] and the R0 of SARS-CoV-2 is 2.2 [10] [23]. This means that SARS$\mathrm{CoV}-2$ is nearly twice as contagious as Influenza. It is expected that the number of cases of COVID-19 will continue to trend upwards in the coming months. Early identification of patients with flu-like symptoms with recent travel to countries endemic with the virus or exposure to others infected with the virus is critical for detection, treatment of illness, and prevention of spread. There is no cure for COVID-19, only supportive therapy for presenting symptoms. There is understandable concern regarding the mortality rate and how easily contagious COVID-19 is when compared to influenza. Efforts must continue to be made to reduce spread. Much research is still needed regarding the origins of SARS-CoV-2.

\section{Conclusion}

Its mode of transmission, treatments, and an effective vaccine.

\section{References}

1. Regan H, Renton A, John T (2020) Global death toll rises to 2,458 as Hubei province in China reports more deaths. CNN.

2. (2020) Secretary Azar Declares Public Health Emergency for the United States for 2019 Novel Coronavirus. US Department of Health and Human Services.

3. Cui J, F Li, ZL Shi (2019) Origin and evolution of pathogenic coronaviruses. Nat Rev Microbiol 17(3): 181-192. 
4. Schoeman D, BC Fielding (2019) Coronavirus envelope protein: current knowledge. Virology Journal 16(1): 69

5. Chang, Minggui Lin, Lai Wei, Lixin Xie, Guangfa Zhu, et al. (2020) Epidemiologic and Clinical Characteristics of Novel Coronavirus Infections Involving 13 Patients Outside Wuhan, China. JAMA.

6. Song Z, Yanfeng Xu, Linlin Bao, Ling Zhang, Pin Yu, et al. (2019) From SARS to MERS, Thrusting Coronaviruses into the Spotlight. Viruses 11(1).

7. Wan Y, Jian Shang, Rachel Graham, Ralph S Baric, Fang Li (2020) Receptor recognition by a novel coronavirus from Wuhan: An analysis based on decade-long structural studies of SARS. J Virol.

8. Lu R, Xiang Zhao, Juan Li, Peihua Niu, Bo Yang, et al. (2020) Genomic characterization and epidemiology of 2019 novel coronavirus: implications for virus origins and receptor binding. The Lancet 395(10224): 565-574

9. Hoffmann M, Hannah Kleine Weber, Nadine Krüger, Marcel Müller, Christian Drosten, et al. (2020) The novel coronavirus 2019 (2019$\mathrm{nCoV}$ ) uses the SARS-coronavirus receptor ACE2 and the cellular protease TMPRSS2 for entry into target cells. bioRxiv.

10. Wang D, Bo Hu, Chang Hu, et al. (2020) Clinical Characteristics of 138 Hospitalized Patients With 2019 Novel Coronavirus-Infected Pneumonia in Wuhan, China. JAMA

11. Huang C, Yeming Wang, Xingwang Li, Lili Ren, Jianping Zhao, et al. (2020) Clinical features of patients infected with 2019 novel coronavirus in Wuhan, China. Lancet 395(10223): 497-506.

12. (2020) Scientists fear coronavirus spread in countries least able to contain it. Nature.

13. Chan JF W, Shuofeng Yuan, Kin-Hang Kok, Kelvin Kai-Wang To, Hin Chu, et al. (2020) A familial cluster of pneumonia associated with the 2019 novel coronavirus indicating person-to-person transmission: a study of a family cluster. Lancet 395(10223): 514-523.
14. Rothe C, Mirjam Schunk, Peter Sothmann, Gisela Bretzel, Guenter Froeschl, et al. (2020) Transmission of 2019-nCoV Infection from an Asymptomatic Contact in Germany. New England Journal of Medicine.

15. Zhang H, Zijian Kang, Haiyi Gong, Da Xu, Jing Wang, et al. (2020) The digestive system is a potential route of 2019-nCov infection: a bioinformatics analysis based on single-cell transcriptomes. bioRxiv.

16. (2020) Centers for Disease Control and Prevention Symptoms, Atlanta, GA. US Department of Health and Human Services. USA.

17. (2020) Centers for Disease Control and Prevention, CDC Tests for COVID-19. Atlanta, GA. US Department of Health and Human Services, USA.

18. Chung M, Adam Bernheim, Xueyan Mei, Ning Zhang, Mingqian Huang, et al. (2020) CT Imaging Features of 2019 Novel Coronavirus (2019-nCoV). Radiology. 0(0): 200230.

19. (2020) Centers for Disease Control and Prevention, Prevention \& Treatment. Atlanta, GA. US Department of Health and Human Services, USA

20. (2020) Centers for Disease Control and Prevention, Weekly U.S. Influenza Surveillance. Atlanta, GA. US Department of Health and Human Services, USA.

21. Nedelman M, Tinker B (2020) CDC has confirmed 35 cases of a novel coronavirus in the US. CNN.

22. Coburn BJ, BG Wagner, S Blower (2009) Modeling influenza epidemics and pandemics: insights into the future of swine flu (H1N1). BMC Med 7: 30 .

23. Li Q, M Med, Xuhua Guan, Peng Wu, Xiaoye Wang, et al. (2020) Early Transmission Dynamics in Wuhan, China, of Novel Coronavirus-Infected Pneumonia. New England Journal of Medicine. 